\section{OPEN ACCESS}

Edited by:

Horolma Pamjav,

Hungarian Institute for Forensic

Sciences, Hungary

Reviewed by:

Balazs Egyed,

Eötvös Loránd University, Hungary

Jatupol Kampuansai,

Chiang Mai University, Thailand

*Correspondence:

Irene Cardinali

cardinali_irene@libero.it

Alessandro Achilli

alessandro.achilli@unipv.it

${ }^{\dagger}$ These authors have contributed equally to this work and share first authorship

Specialty section:

This article was submitted to Evolutionary and Population Genetics,

a section of the journal

Frontiers in Genetics

Received: 21 November 2021

Accepted: 14 December 2021

Published: 06 January 2022

Citation:

Cardinali I, Bodner M, Capodiferro MR,

Amory C, Rambaldi Migliore N, Gomez EJ, Myagmar E, Dashzeveg T, Carano F. Woodward SR, Parson W, Perego UA, Lancioni $H$ and Achilli $A$ (2022) Mitochondrial DNA Footprints from Western Eurasia in Modern Mongolia.

Front. Genet. 12:819337. doi: 10.3389/fgene.2021.819337

\title{
Mitochondrial DNA Footprints from Western Eurasia in Modern Mongolia
}

\author{
Irene Cardinali ${ }^{1 * t}$, Martin Bodner ${ }^{2 t}$, Marco Rosario Capodiferro ${ }^{3 \dagger}$, Christina Amory ${ }^{2}$, \\ Nicola Rambaldi Migliore ${ }^{3}$, Edgar J. Gomez ${ }^{4,5}$, Erdene Myagmar ${ }^{6}$, Tumen Dashzeveg ${ }^{6}$, \\ Francesco Carano ${ }^{7}$, Scott R. Woodward ${ }^{4}$, Walther Parson ${ }^{2,8}$, Ugo A. Perego ${ }^{3,4,9}$, \\ Hovirag Lancioni ${ }^{1}$ and Alessandro Achilli ${ }^{3 *}$
}

${ }^{1}$ Department of Chemistry, Biology and Biotechnology, University of Perugia, Perugia, Italy, ${ }^{2}$ Institute of Legal Medicine, Medical University of Innsbruck, Innsbruck, Austria, ${ }^{3}$ Department of Biology and Biotechnology "L. Spallanzani”, University of Pavia, Pavia, Italy, ${ }^{4}$ Sorenson Molecular Genealogy Foundation, Salt Lake City, UT, United States, ${ }^{5}$ FamilySearch Int., Salt Lake City, UT, United States, ${ }^{6}$ Department of Anthropology and Archaeology, National University of Mongolia, Ulaanbaatar, Mongolia, ${ }^{7}$ Department of Medical and Surgical Sciences, University of Bologna, Bologna, Italy, ${ }^{8}$ Forensic Science Program, The Pennsylvania State University, State College, PA, United States, ${ }^{9}$ Department of Math and Science, Southeastern Community College, Burlington, IA, United States

Mongolia is located in a strategic position at the eastern edge of the Eurasian Steppe. Nomadic populations moved across this wide area for millennia before developing more sedentary communities, extended empires, and complex trading networks, which connected western Eurasia and eastern Asia until the late Medieval period. We provided a fine-grained portrait of the mitochondrial DNA (mtDNA) variation observed in present-day Mongolians and capable of revealing gene flows and other demographic processes that took place in Inner Asia, as well as in western Eurasia. The analyses of a novel dataset $(N=2,420)$ of $m t D N A s$ highlighted a clear matrilineal differentiation within the country due to a mixture of haplotypes with eastern Asian (EAs) and western Eurasian (WEu) origins, which were differentially lost and preserved. In a wider genetic context, the prevalent EAs contribution, larger in eastern and central Mongolian regions, revealed continuous connections with neighboring Asian populations until recent times, as attested by the geographically restricted haplotype-sharing likely facilitated by the Genghis Khan's so-called Pax Mongolica. The genetic history beyond the WEu haplogroups, notably detectable on both sides of Mongolia, was more difficult to explain. For this reason, we moved to the analysis of entire mitogenomes $(N=147)$. Although it was not completely possible to identify specific lineages that evolved in situ, two major changes in the effective (female) population size were reconstructed. The more recent one, which began during the late Pleistocene glacial period and became steeper in the early Holocene, was probably the outcome of demographic events connected to western Eurasia. The Neolithic growth could be easily explained by the diffusion of dairy pastoralism, as already proposed, while the late glacial increase indicates, for the first time, a genetic connection with western Eurasian refuges, as supported by the unusual high frequency and internal sub-structure in Mongolia of haplogroup $\mathrm{H} 1$, a well-known post-glacial marker in Europe. Bronze Age events, without a significant demographic impact, might explain the age of some mtDNA haplogroups. Finally, a diachronic comparison with available ancient mtDNAs made it 
possible to link six mitochondrial lineages of present-day Mongolians to the timeframe and geographic path of the Silk Route.

Keywords: Eurasian Steppe, Inner Asia, Mongolia genetic history, modern mitogenomes, mitochondrial DNA phylogeny, mtDNA haplogroups

\section{INTRODUCTION}

The Eurasian Steppe stretches from Europe to Inner Asia and represents an important crossroad in human history, characterized by migrations and admixtures of culturally and genetically distinct populations (Palstra et al., 2015). Mongolia covers most of the Eastern Steppes. Nowadays, it is a presidential republic divided into 21 provinces (aimags) and one provincial municipality (Ulaanbaatar); most of the population (71\%) lives in urban centers, while the remaining $29 \%$, often tied to nomadic lifestyles, lives in rural areas.

Archaeological evidence (Kovalev and Erdenebaatar, 2009; Wilkin et al., 2021) and genetic studies provided the first information on the complex Mongolian past (Cavalli-Sforza et al., 1994; Comas et al., 1998; Yao et al., 2004; Yang et al., 2008; Yunusbayev et al., 2015; Pugach et al., 2016; Bai et al., 2018). As in another population context (Achilli et al., 2018), archaeogenomics unveiled further details on the emerging scenario of admixture between Eastern and Western Eurasians for the origin of the Central Asian populations, with distinct westeast genetic gradients between different western and eastern Eurasian groups (Jeong et al., 2019; Narasimhan et al., 2019; Ning et al., 2021). In particular, recent analyses of ancient genomes spanning from 6000 before the common era (BCE) to present days revealed at least four ancestral sources that arose in Mongolia through the Neolithic. Two were identified in preBronze Age individuals from northeastern and northern Mongolia and are associated to hunter-gatherer populations from northeast Asia and northern Eurasia, respectively; the third was connected with the Afanasievo culture, an eastward extension of the Yamnaya culture from the Pontic-Caspian steppe (ca. 3300-2200 BCE), which probably introduced dairying practices to the region (ca. $3000 \mathrm{BCE}$ ) and was later followed or replaced by the Chemurchek culture (2750-1900 $\mathrm{BCE})$. A genetic mixture of Yamnaya pastoralists and European farmers, the fourth source, appeared ca. 1400 BCE (Jeong et al., 2020; Wang C. C. et al., 2021). During the Middle and Late Bronze Age (ca. 1900-900 BCE), ruminant dairying characterized by intensive nomadic herding without farming was widespread, leading to the development of large-scale polities since the late first millennium BCE. The Xiongnu was the first of different historically documented dynasties and empires, founded by pastoralists in the Early and Late Medieval periods such as Xianbei, Türk, Uyghur, Khitan, and Mongol. During those centuries Mongolia and the Eurasian Steppe represented an important crossroad through the notorious Silk Road (founded by the Chinese Han dynasty in $130 \mathrm{BCE}$ ) that played a major role in the economic, demographic and cultural processes shaping the history of several Eurasian populations (Comas et al., 1998). The Mongol empire arose in the late 12th century CE when the chieftain Temüjin took the title of Genghis Khan ("Universal Ruler"). At its peak (1206-1368 CE), the empire stretched from present-day Poland in the west to Korea in the east, and from Siberia in the north to the Gulf of Oman and Vietnam in the south, covering approximately $22 \%$ of Earth's total land and with a population of over 100 million people. At the beginning, Genghis Khan used to destroy most infrastructures along the Silk Route, but eventually he decided to adopt a politics of supporting and facilitating commercial and cultural exchanges between regions under his dominion (Liu, 2010). The empire allowed the establishment of the Pax Mongolica (1280-1360 CE), indicating a pacific and flourishing period characterized by commercial, cultural, religious, and scientific exchanges between western and eastern populations, including trades between nomadic groups and urban centers (Köstenbauer, 2017). The historical stability under Genghis Khan's rule is also supported when comparing genetic profiles of ancient Mongols with contemporary Mongolians (Zerjal et al., 2003; Bai et al., 2018).

Mitochondrial DNA (mtDNA) provided several pieces of information. Evidence deriving from the mtDNA haplogroups shared between Afanasievo and Yamnaya people supports an eastward migration from the Pontic-Caspian steppes (Allentoft et al., 2015; Narasimhan et al., 2019). The presence of a U5a1 mitochondrial haplotype in an Eneolithic grave, dated at ca. 3000 BCE and associated with the Afanasievo archaeological culture in the Khangai Mountains, attested the presence of people with "western" origin in the east of the Altai Mountains before the Bronze Age (Rogers et al., 2020), in contrast to what was previously proposed (Ricaut et al., 2004a; Ricaut et al., 2004b; Lalueza-Fox et al., 2004; Chikisheva et al., 2007; Keyser et al., 2009; González-Ruiz et al., 2012; Wang C. C. et al., 2021). To further investigate the impact and legacy of mitochondrial lineages with eastern and western origins on the gene pool of modern Mongolian populations, we analyzed the mtDNA profiles of 2,420 individuals with a last known terminal maternal ancestor (TMA) from one of the 20 different Mongolian provinces.

\section{MATERIALS AND METHODS}

\section{Sample Collection and DNA Extraction}

A total of 2,420 biological samples belonging to unrelated subjects with a Mongolian TMA were collected in different areas of Mongolia, using $10 \mathrm{ml}$ of commercially available mint-flavored mouthwash. Pedigree charts and informed consents were obtained from all participants. The samples were collected in 20 (out of 21) Mongolian provinces: Arkhangai $(n=4)$, Bayankhongor $(n=2)$, Bayan-Ölgii $(n=216)$, Bulgan $(n=5)$, 
Darkhan-Uul $(n=1)$, Dornod $(n=370)$, Dornogovi $(n=26)$, Dundgovi $(n=1)$, Govi-Altai $(n=8)$, Khentii $(n=132)$, Khovd ( $n=429)$, Khövsgöl $(n=307)$, Ömnögovi $(n=2)$, Övörkhangai $(n=8)$, Selenge $(n=4)$, Sükhbaatar $(\mathrm{n}=246)$, Töv $(n=10)$, Ulaanbaatar $(n=2)$, Uvs $(n=132)$, Zavkhan $(n=167)$; the remaining samples $(n=348)$ belonged to individuals who did not provide province information and therefore were associated to an "unspecified" group. Provinces with less than 30 individuals were grouped into three geographic macro-areas by considering their geographic position, biome, and orography: "Gobi Desert" (Bayankhongor, Dornogovi, Dundgovi, Govi-Altai and Ömnögovi), "Khangai Mountains" (Arkhangai and Övörkhangai), "Near Ulaanbaatar" (Bulgan, Darkhan-Uul, Selenge, Töv and Ulaanbaatar) (Supplementary Table S1).

\section{Mitochondrial DNA Control Region Analysis}

DNA extraction and mtDNA control-region sequencing were performed as in Perego et al. (2012).

All resulting sequences have been deposited in GenBank under accession numbers OL632312-OL634731 and are also available in the EMPOP mtDNA population database (https://empop.online/) under accession number EMP00853. A total of 2,133 haplotypes encompassed the entire mitochondrial control region (CR, 1122 bps from np 16024 to $\mathrm{np}$ 576), while 2,335 haplotypes encompassed at least the HVS1 segment (nps 16024-16365). The sequences were aligned to the revised Cambridge Reference Sequence (rCRS; NC_012920.1) (Andrews et al., 1999) using Sequencher 5.10 (Gene Codes Corporation), in order to visualize electropherograms and identify and register any mutational differences. All samples were classified into haplogroups according to their respective mutational motifs by referring to PhyloTree build 17 (van Oven and Kayser, 2009). Considering the large number of maternal lineages identified in our study we grouped each of them into 18 macro-haplogroups (Supplementary Table S2).

Several mtDNA sequence variation parameters were estimated by using DnaSP 5.1 software (Librado and Rozas, 2009). Nucleotide diversity ( $\pi$ or $\mathrm{Pi}$ ) and haplotype diversity (Hd) were plotted with Tableau 2021tbl 2021.3 onto Mongolian geographic map. In order to graphically display and summarize the mitogenetic relationships among the analyzed individuals, Principal Component Analyses (PCA) were performed using prcomp () from the stats R package ( $\mathrm{R}$ Core Team, 2021) or the Excel software implemented by XLSTAT. Macro-haplogroup frequencies were used as input data. In intraMongolia analyses, the Khangai Mountains and the cosmopolitan "Near Ulaanbaatar" macro-area were excluded due to the low number $(<30)$ of individuals in each of them.

The Mongolian maternal gene pool was further compared with a Eurasian dataset encompassing 546 bps of the control region (nps 16024-16569) obtained from 30,400 individuals from 69 countries/geographic areas (Supplementary Table S3). Four population groups outside Mongolia with less than 30 individuals were excluded from these analyses. The final dataset (for a total of 32,486 sequences including 2,133 Mongolian CR sequences from this study) was aligned with MEGAX (Kumar et al., 2018). The genetic distance between groups was also calculated with
MEGAX using the p-distance (proportion of nucleotides at which two sequences being compared are different). The obtained distance matrix was used to construct a multidimensional scaling (MDS) using the $\mathrm{R}$ function cmdscale () (R Core Team, 2021). The heteroplasmic bases were converted to Ns with DNAsp 6 (Rozas et al., 2017), before calculating the haplotype sharing with Arlequin (Excoffier and Lischer, 2010). The ratio of haplotype sharing was calculated for each population pair by dividing the number of shared haplotypes by the total haplotypes in each paired group. The pairwise haplotype sharing ratio was also calculated separately for haplotypes belonging to eastern Asia (EAs) and western Eurasian (WEu) haplogroups.

\section{Complete Mitogenome Analysis}

The entire mitogenome sequences of 147 individuals, representative of different macro-haplogroups, were obtained (Supplementary Table S4) by using the Ion Torrent Personal Genome Machine (PGM) and following manufacturers' protocols. The entire mtDNA molecule was amplified with the HID-Ion Ampliseq Mitochondrial Library Preparation; then the template-positive Ion PGM Hi-Q Ion Sphere Particles were prepared with Ion OneTouch 200 Template Kit v2 and sequenced with the Ion PGM Hi-Q Sequencing Kit chemistry on an Ion 318 v2 chip using a multiplexing approach (Parson et al., 2013; Strobl et al., 2018).

The IGV (Integrative Genomics Viewer) software (Thorvaldsdóttir et al., 2013) was used to visualize the BAM files (aligned to the rCRS and produced by the sequencing machine aligning software) and to verify or to search for specific mutational differences throughout the entire mitochondrial genome. Ambiguous positions were analyzed by Sanger-type sequencing until clarity was reached. The quality of mitogenome sequences was checked through SAM2 on EMPOP (Parson and Dür, 2007; Huber et al., 2018), and all samples were classified into haplogroups according to PhyloTree build 17 (http://www.phylotree.org/) (van Oven and Kayser, 2009) using both Haplogrep 2.0 (Weissensteiner et al., 2016) and SAM2 on EMPOP. A few discrepancies using the two approaches were expected, as explained in Dür et al. (2021). However, these differences do not affect the outcomes of the present work. All control-region haplotypes were confirmed by the mitogenome sequences.

All complete mitogenomes $(\mathrm{N}=147)$ are available in GenBank under accession numbers OL619795-OL619941 and in the EMPOP mtDNA population database (https://empop.online/) under accession number EMP00853.

The evolutionary relationships among our modern haplotypes were visualized through the construction of a most parsimonious (MP) tree, built with an updated version of mtPhyl v.5.003 and checked with MEGAX software. One published L3 sequence (accession number DQ341081) was used as outgroup to reconstruct time estimates and demographic trends in BEAST v2.6.6 (Bouckaert et al., 2019), as previously reported (Modi et al., 2020; Capodiferro et al., 2021). 95\% of High Posterior Densities (HPD) were plotted for haplogroups younger than 20 thousand years ago (kya). A total of 693 published ancient mitogenomes from 


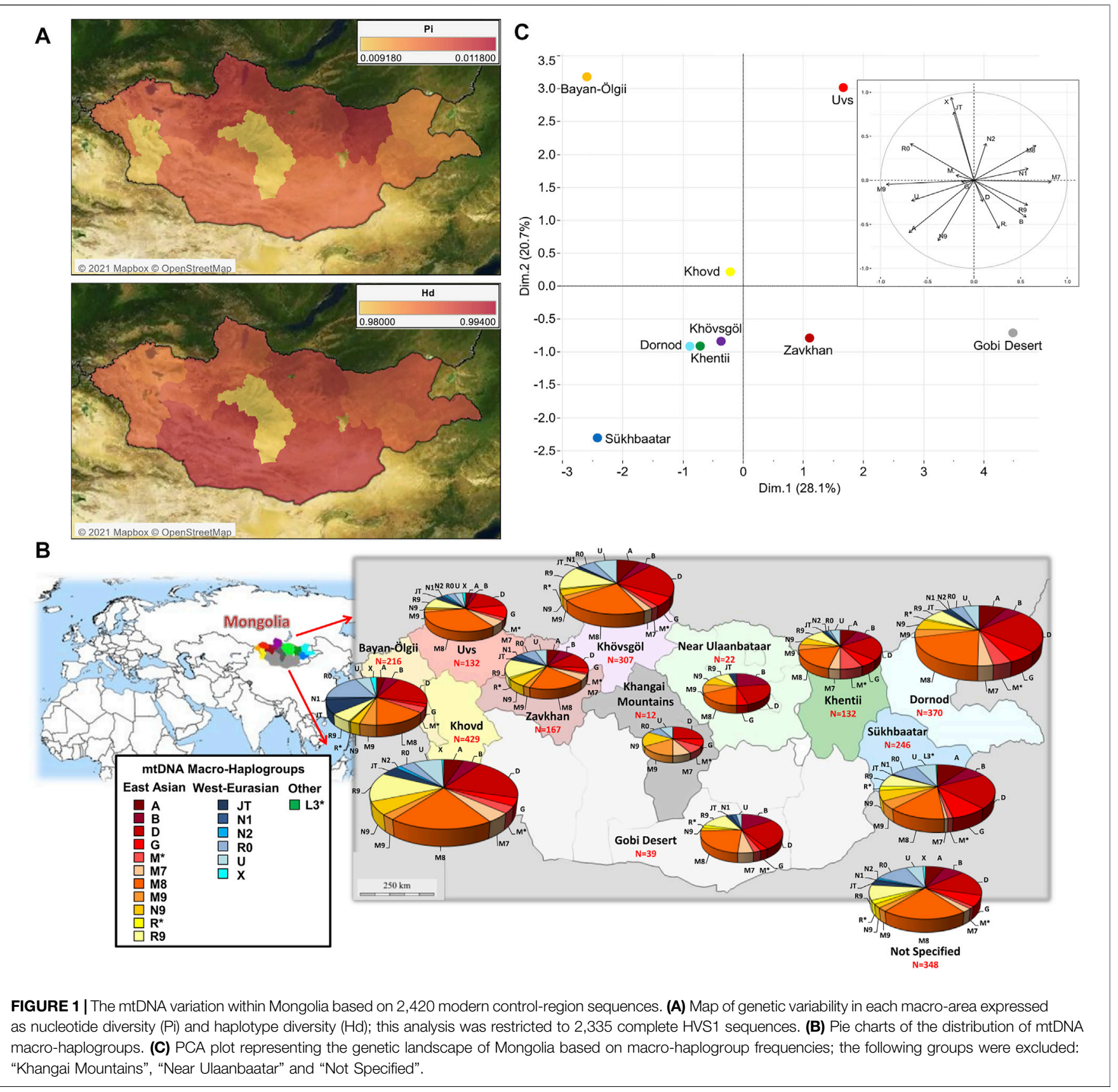

different Eurasian regions (including 25 excavated in Mongolia) were also analyzed taking into account haplogroup classification, age and location of the remains (Supplementary Table S5).

\section{RESULTS}

\section{The Mitochondrial DNA Variation Within Mongolia}

The concomitant analysis of 2,335 HVS1 sequences from modern Mongolians identified 160 polymorphic sites (excluding gaps and ambiguous sites) with a Nei's nucleotide diversity ( $\pi$ or Pi) of
0.00809 , and a very high haplotype diversity $(\mathrm{Hd}=0.986)$. This genetic diversity is heterogeneously distributed within Mongolia (Figure 1A). The "Near Ulaanbaatar" macro-area is characterized by high diversity values, probably due to very recent migrations towards regions around the capital. On the other hand, isolation could better explain the very low diversity in the Khangai Mountains, even if a sample bias due to the low number of individuals $(\mathrm{N}=12)$ cannot be excluded. Interestingly, the Khovd region shows an average number of different haplotypes but a very low nucleotide diversity, which might indicate that people of this western area stretched between the Altai Mountains and the Gobi Desert brought different haplotypes in this area only 

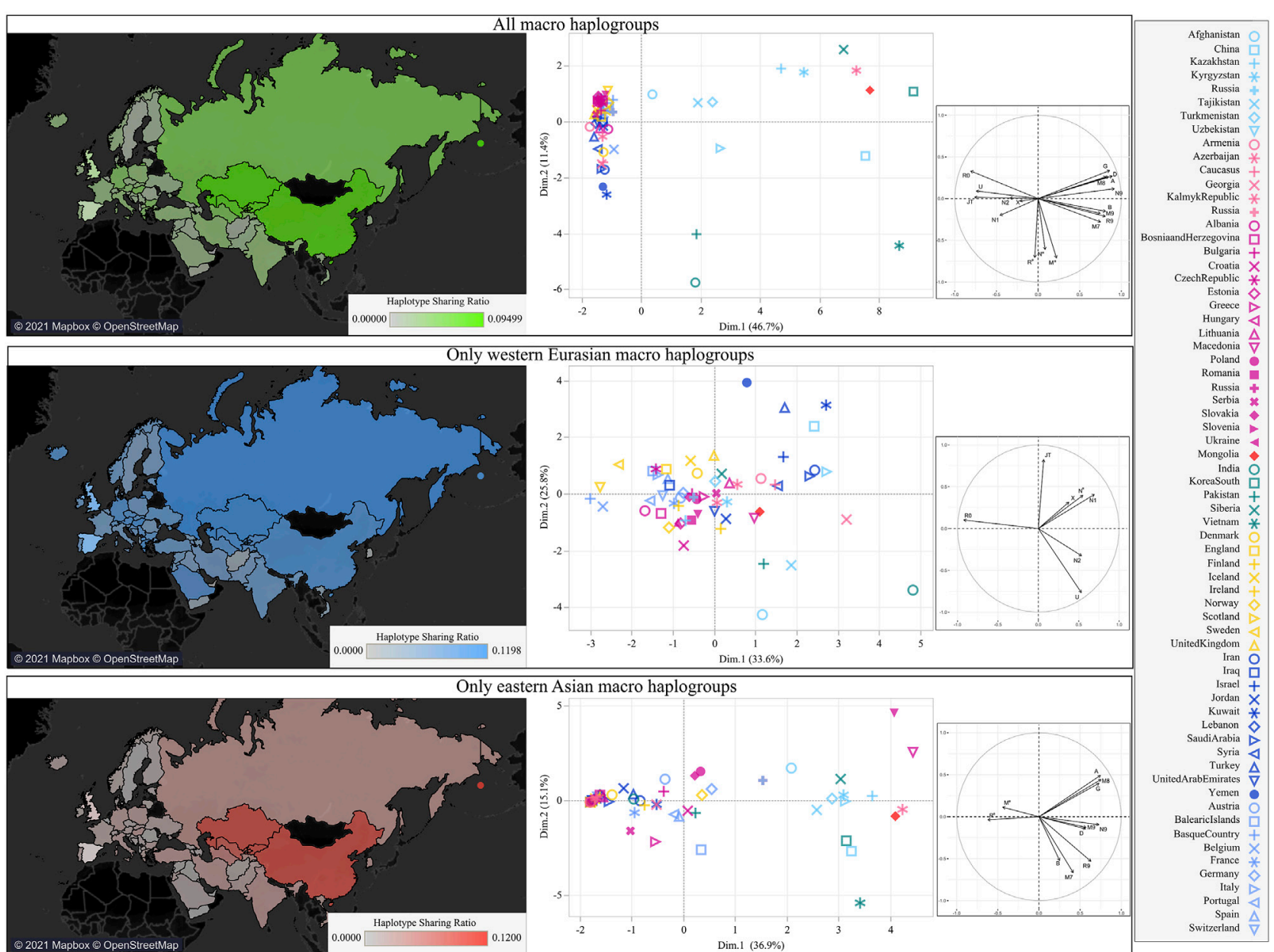

FIGURE 2 | The mtDNA variation of Mongolia in the Eurasian context. Heatmaps based on haplotype sharing between Mongolia and other Eurasian populations are shown on the left. PCA plots based on macro-haplogroup frequencies are shown on the right. All lineages were included in the top panel, the differential contributions of WEu and EAs lineages were explored in the middle and lower panel, respectively.

recently, and their mitogenomes did not yet differentiate from each other.

The overall 2,420 Mongolian mtDNAs were classified into different 413 lineages and sub-lineages, ultimately grouped into 18 macro-haplogroups (A, B, D, G, JT, L3*, M $^{\star}, \mathrm{M} 7, \mathrm{M} 8, \mathrm{M} 9, \mathrm{~N} 1$, N2, N9, R*, R0, R9, U, and X; Supplementary Table S2). The macro-haplogroup distribution across the country clearly shows a differential contribution of haplogroups with two distinct geographic origins (Figure 1B; Supplementary Table S1). As expected, most mtDNAs (1,987 out of 2,420: $82.1 \%$ ) belong to eastern Asia (EAs) haplogroups with a notable incidence of $\mathrm{C}$ (19.6\%) and D4 (19.8\%) and higher frequencies in the eastern part of Mongolia. In the west, the presence of western Eurasian (WEu) haplogroups is significant $(21.7 \%)$ and tends to decrease eastwards, but with the lowest occurrences in central regions. The most represented WEu haplogroup is $H(6.5 \%)$, thus confirming the results obtained in previous studies on Inner Asia (Comas et al., 1998; Wells et al., 2001; Quintana-Murci et al., 2004; Derenko et al., 2014; Lan et al., 2019; Chen et al., 2020; Wang W. et al., 2021; Keyser et al., 2021). A geographic differentiation is clear in the PCA that represents the mtDNA genetic landscape of Mongolia (Figure 1C). The PC2 separates the westernmost regions, Bayan-Ölgii, Khovd and Uvs, due to the high contribution of the typical WEu lineages JT, N2, R0 and X. The northern regions (Dornod, Khentii and Khövsgöl) cluster together in the middle of the plot, while PC1 pushes the southern regions of Gobi Desert and Sükhbaatar apart from each other due to the differential distribution of typical EAs lineages.

\section{The Mitochondrial DNA Variation of Mongolia in the Eurasian Context}

Considering the various mtDNA contributions to different regions within the country, we evaluated the Mongolian mitochondrial gene pool in the Eurasian context through a MDS plot based on genetic distances, detecting an outlier behavior (Supplementary Figure S1). This peculiarity was further investigated through the analysis of haplotype sharing and by building a Eurasian PCA based on macro-haplogroup frequencies (Figure 2). The map based on haplotype sharing shows a greater proximity to 


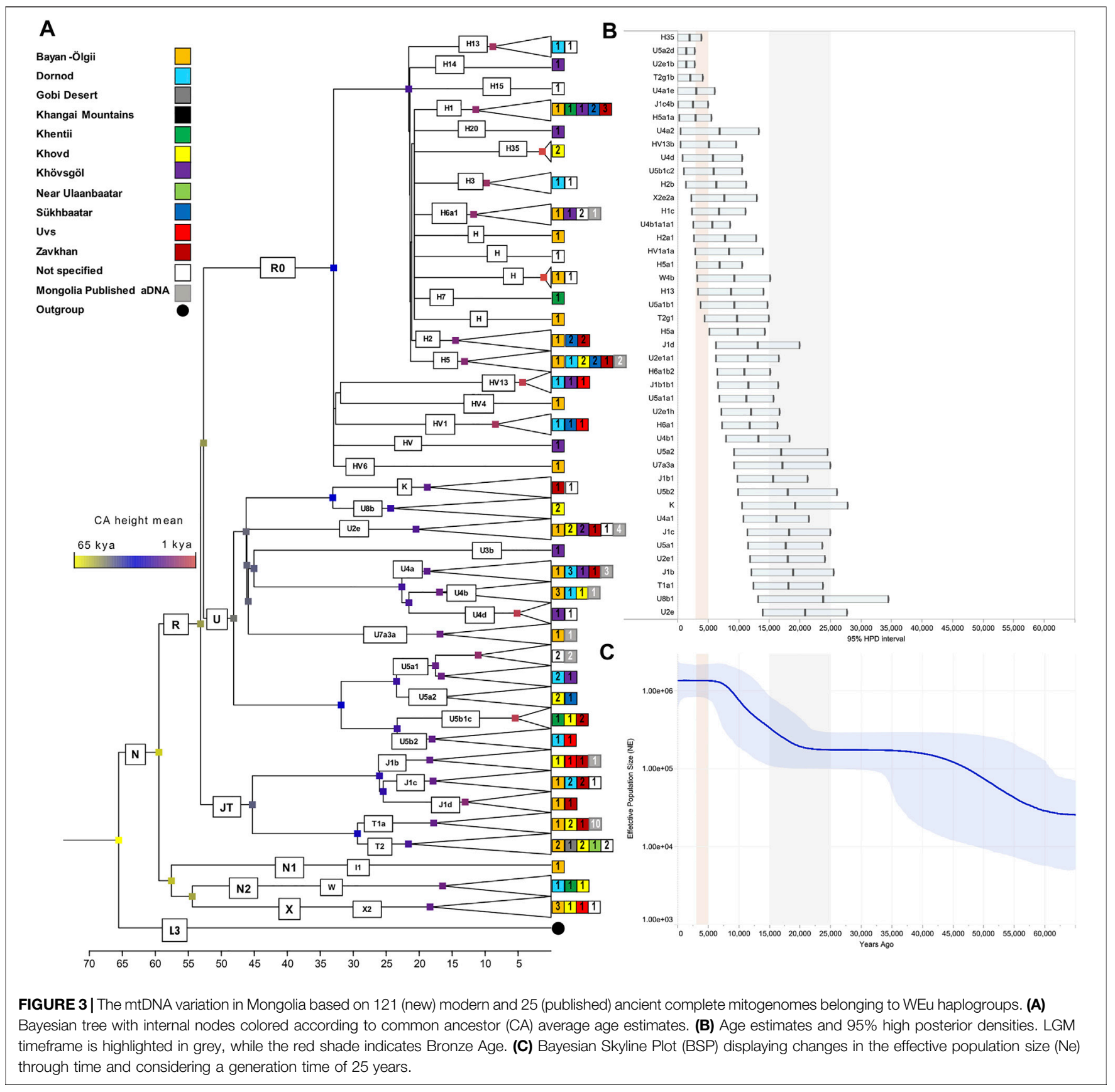

surrounding Asian populations. When we tried to differentiate eastern and western contributions, the legacy of WEu haplogroups was less marked and more widespread. Therefore, it is apparent that the genetic relationships with neighboring populations were mostly driven by EAs lineages and the higher haplotype sharing suggests continuous interactions.

\section{The Mitochondrial DNA Variation in Mongolia Based on Complete Mitogenomes} To deepen the understanding of mtDNA peculiarities of Mongolians, we extended the analysis to the maximum level of resolution. A total of 147 complete mitogenomes were obtained, including $26 \mathrm{mtDNAs}$ representative of EAs lineages $\left(B, C, D, F, M^{*}, R 1, R 2, R 11\right)$ and 121 belonging to WEu haplogroups (H, HV, I, J, K, T, U, W, X) (Supplementary Figure S2; Supplementary Table S4). Through the phylogenetic analysis of our complete mitogenomes, we identified two novel mtDNA sub-branches of haplogroups $\mathrm{HV}$ and U5. Three different HV haplotypes from the provinces of Dornod, Khövsgöl and Uvs showed common mutational motifs both in coding (at nps 1654, 9377 and 11152) and control regions (at nps 16184 and 16291) in addition to HV13b motif, thus allowing us to classify these 


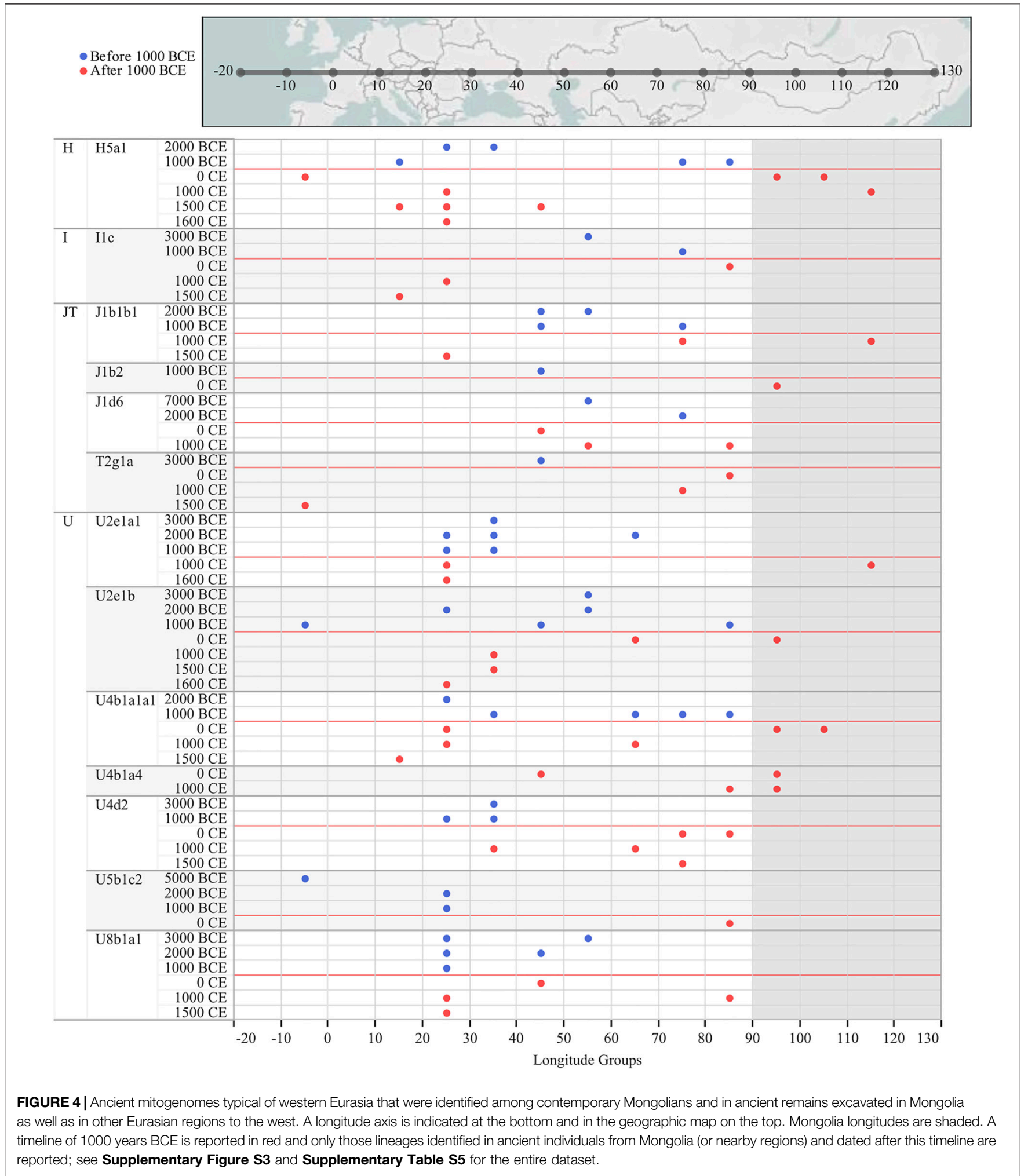

sequences into a postulated haplogroup HV13b1. Four Mongolian individuals from Khentii, Khovd and Zavkhan presenting three different haplotypes were classified as U5b1c2, due to a common transition at np 9110. The entire haplogroup H1 was represented in our control-region dataset with a frequency three times higher $(\sim 3 \%)$ than the value reported in literature for Inner Asia (Ottoni et al., 2010) and through complete mitogenome analysis, we identified different 
sub-clades: $\mathrm{H} 1 \mathrm{~b}$ and $\mathrm{H} 1 \mathrm{c}$, previously found in Asia as well as in Europe, and $\mathrm{H} 1 \mathrm{j}$, that is very uncommon in the Asian continent.

In order to provide a timeframe to the mtDNA inputs from the west, we focused on the WEu mitogenomes providing Bayesian coalescence ages of internal nodes. Most of these lineages, with a western Eurasian origin but now also identified in Mongolia, coalesced during and soon after the last glacial maximum (LGM, 25-15 kya; purple nodes) (Figures 3A,B). The BSP describes a demographic trend with two major increases of the effective population size $(\mathrm{Ne}$; Figure 3C). The first one between 60 and 45 kya likely reflects the initial increase of $\mathrm{Ne}$ due to the initial settlement of Eurasia by modern humans during the Pleistocene after the Out-of-Africa exit. Another major increment of $\mathrm{Ne}$ seems to be characterized by two steps: the first one started in the late glacial period $\sim 18-15 \mathrm{kya}$, while the second took place in early Holocene and was probably facilitated by ecological changes associated with the Holocene Climatic Optimum (HCO; 10-6 kya) (An et al., 2008; Orkhonselenge et al., 2018). When considering the $95 \%$ HPD, we noticed that some lineages started to coalesce in the early Bronze Age ( $\sim$ kya). They were probably carried by nomadic steppe populations but did not contribute significantly to the Ne. Finally, a very few haplogroups originated in more recent times ( $<3 \mathrm{kya})$ and could be linked to historical events. The latter timeframe cannot be accurately described through coalescent estimates taking into account that mitogenomes accumulate one mutation in more than two thousand years on average (Soares et al., 2009; Posth et al., 2016). Therefore, we tried to temporally and geographically reconstruct possible routes marked by the WEu lineages of modern Mongolians, by building a map with the ancient mitogenomes belonging to these lineages that were identified in ancient remains excavated in Mongolia (and nearby regions) as well as in other Eurasian regions to the west. Considering a post-Bronze Age timeline of one thousand years BCE, it is clear that the majority of these WEu lineages were already present in Mongolia in prehistoric times (Supplementary Figure S3). However, other WEu lineages (H5a1, I1c, J1b1b1, J1b2, J1d6, T2g1a, U2e1a1, U2e1b, U4b1a1a1, U4b1a4, U4d2, U5b1c2, U8b1a1) reached Mongolia (or nearby regions) after 1000 BCE (Figure 4) after appearing in areas 20-40 degrees of longitude to the west.

\section{DISCUSSION}

Mongolia is one of the most sparsely populated countries in the world, but complex population interactions occurred across this Eastern Steppe region over the past several millennia (Schurr and Pipes, 2011). Here we characterized the mtDNA of 2,420 modern individuals with a TMA from Mongolia. Our analyses showed a high mitochondrial variation that is heterogeneously distributed across the country. The higher diversity values are present in the northern regions with a dramatic increase in the cosmopolitan area near the capital Ulaanbaatar. If the first finding agrees with reported paleogenomic data (Jeong et al., 2019), the peculiarity of the area around the capital could be better explained by more recent migrations that probably wiped out the original mtDNA gene pool. Other populations probably remained more isolated due to geographic barriers (mountains and desert), which reduced the number of different mitogenomes since ancient times (e.g., in the Khangai Mountains) or only recently (e.g., in the Khovd region).

The majority of mtDNAs belong to haplogroups typical of eastern Asian populations whose frequency decreases from eastern to western regions. An opposite pattern could be observed for those lineages characteristic of western Eurasia. Overall, both EAs and WEu haplogroups contributed to create the mtDNA differentiation currently detectable in Mongolia, as highlighted by the PCA (Figure 1). The WEu lineages determine the genetic distinction of the three westernmost provinces (Bayan-Ölgii, Khovd and Uvs), mostly due to macro-haplogroups JT, R0 and X. In particular, macrohaplogroup R0 (mostly made of $\mathrm{H}$ mtDNAs, 36.1\%) characterizes the outlier position of people living in the Bayan-Ölgii province, which encompasses the Altai Mountains. The Altai Mountains have initially been considered a genetic barrier to gene flows from the west until the recent discovery of ancient people with a WEu mtDNA living on the Mongol Steppe east of the Altai Mountains before the Bronze Age (Rogers et al., 2020). Different EAs lineages distinguish the southern regions, while the northeastern provinces (Dornod, Khentii, Khövsgöl and Sükhbaatar) cluster together, separately from the others, and are characterized by a high number of different mitogenomes that arrived mostly from the surrounding eastern Asian countries. Actually, genetic closeness and continuous interactions of Mongolia with neighboring populations are witnessed by the shared haplotypes of typical EAs lineages. During Early and Late Medieval time, these interactions across the east Asian Steppe were probably facilitated by a series of organized and highly influential nomadic empires, which had a major impact on the demography and geopolitics of Eurasia until the fall of the Mongol Empire (Jeong et al., 2020). A different pattern, more homogeneous and widespread, has been observed when considering shared haplotypes belonging to WEu lineages without pointing to any apparent connection. Therefore, we pushed the analysis to the highest level of resolution, by considering the information hidden in complete mitogenomes and reconstructing and dating the phylogenetic tree of western Eurasian haplogroups found in Mongolia. The ages of some lineages fall in late and postglacial times and the demographic analysis highlighted a significant increase of mtDNA lineages and population size that started right after the LGM and became steeper during the HCO, probably marking two different demographic events. The first reflects post-glacial re-populations from glacial refuges in western Eurasia, as testified by the haplotype sharing with contemporary populations from Europe and the Balkans peninsula (a well-known refuge area during 
LGM), and the high frequency of haplogroup H1, which was indicated as a genetic marker of the post-glacial expansions from western European refuge areas (Achilli et al., 2004). A post-glacial expansion in eastern Asia was already proved for another mtDNA post-glacial marker, haplogroup U5b (Achilli et al., 2005). A later expansion can be probably connected to the climatic amelioration of the early Holocene that was accompanied by the development of farming and pastoralism and more sedentary communities. A mixed ancestry between Yamnaya and European farmers was recently identified by analyzing ancient Bronze Age Mongolians (Jeong et al., 2020; Wang C. C. et al., 2021). We could not identify sub-branches of $\mathrm{WEu}$ lineages specific to Mongolia. Therefore, most of the WEu lineages detected in modern Mongolians actually evolved in western Eurasia, and the increments of the population size depicted by our BSP might mirror demographic events that took place in regions to the west of Mongolia. The lack of Mongolia-specific sub-branches might also suggest that the WEu lineages arrived in the Eastern Steppe in more recent times. Certainly, the ages of some WEu lineages between 5 and 3 kya could be linked to Bronze Age migrations across the Eurasian steppes that probably involved also the Afanasievo first (ca. 3300-2500 BCE) and later the Sintashta culture (ca. 2100-1800 BCE). Finally, by searching the available database of ancient mitogenomes for WEu lineages identified in our modern Mongolians, we identified 13 different sub-lineages among remains excavated in Mongolia and dated after the Bronze Age. They might testify for small population movements from the west less than 3,000 ya that can be probably related to commercial routes. Actually, the migration path from western Eurasia to Mongolia marked by some of these mitochondrial sub-lineages (H5a1, J1b2, T2g, U2e1b, U4b1a1a1, and U4b1a4) occurred about 2,500 ya, thus temporally and geographically overlapping with the Silk Route, while other sub-haplogroups, such as J1b1b1 and U2ela1, seem to have arrived in Mongolia later.

\section{CONCLUSION}

The gene pool of present-day Mongolians reflects gene flows and demographic processes that occurred over the past several millennia across the Eurasian Steppe, thus representing an important key to reconstruct the genetic history of Inner Asia as well as western Eurasia. The analyses of a large set $(\mathrm{N}=2,420)$ of $\mathrm{mtDNAs}$ allowed us to identify peculiarities of the mitochondrial gene pools of different Mongolian regions. A clear matrilineal differentiation was identified across the country due to the differential contribution of mitochondrial lineages of eastern Asian and western Eurasian origins. The EAs contribution was probably linked to continuous interactions with neighboring regions until present days, presumably including those related to the Mongol Empire expansions (1206-1368 CE). The inputs from the west were more difficult to pinpoint. Therefore, we moved to the analysis of entire mitogenomes $(\mathrm{N}=147)$, which allowed us to date the WEu lineages and to reconstruct demographic trends across time. After the first migration of Paleolithic hunter-gatherers, the major increases of the population size could be linked to post-glacial late Pleistocene expansions and to changes towards a more sedentary lifestyle during the Holocene. However, the lack of Mongolia-specific lineages did not allow to directly study mitogenomes that evolved in situ. A few and more recent events have been also reconstructed through the analysis of modern and ancient mitogenomes, some during the Bronze Age, others in the last three thousand years. As if haplogroup H1 (and its sub-clades) might suggest a direct link between Europe and Mongolia, six sub-lineages identified in ancient mitogenomes perfectly match the timeframe and path of the Silk Route and can be still identified in present-day Mongolians. Finally, rather than finding long-distance traces of the Mongol Empire expansion to the west, we identified continuous and recent (female-mediated) connections with neighboring Eastern Asian populations. The geographically restricted sharing of haplotypes from typical EAs mtDNA lineages might represent an outcome of Genghis Khan's so-called Pax Mongolica still detectable in present-day Mongolians.

\section{DATA AVAILABILITY STATEMENT}

The original contributions presented in the study are publicly available. This data can be found here: OL632312-OL634731 for mtDNA control regions and OL619795-OL619941 for complete mitogenomes.

\section{ETHICS STATEMENT}

The studies involving human participants were reviewed and approved by Western Institutional Review Board (WIRB), Olympia, Washington (United States). The patients/ participants provided their written informed consent to participate in this study.

\section{AUTHOR CONTRIBUTIONS}

UAP and AA conceived the study. EG, TD, EM, SW, and UAP provided samples and genealogical data. IC, MB, MRC, CA, UAP, HL, and AA performed the laboratory experiments. IC, MB, MRC, CA, NRM, UAP, HL, and AA conducted the data analyses. IC, MB, MRC, CA, NRM, and WP performed the data quality control and validation. EG, EM, FC, and WP contributed to data interpretation. IC, MB, MRC, HL, and AA wrote the original draft with inputs from all co-authors. All authors reviewed and approved the final manuscript.

\section{FUNDING}

This research received support from: the National Geographic Society (NGS) grant number HJ-115ER-17 (to 
IC); the Italian Ministry of Education, University and Research (MIUR) for Progetti PRIN 2017 20174BTC4R (to AA) and Dipartimenti di Eccellenza Program (2018-2022) - Department of Biology and Biotechnology "L. Spallanzani," University of Pavia (to MC, NRM, and AA); the University of Pavia-INROAd program (to AA). TD and EM are supported by the Ministry of Education, Culture, Science and Sport of Mongolia (grant \#2018/25) and the Russian Foundation for Basic Research (grant \#18-59-94,020); EM is also supported by the National University of Mongolia (grant number P2020-3955).

\section{REFERENCES}

Achilli, A., Olivieri, A., Semino, O., and Torroni, A. (2018). Ancient Human Genomes-Keys to Understanding Our Past. Science 360 (6392), 964-965. doi:10.1126/science.aat7257

Achilli, A., Rengo, C., Battaglia, V., Pala, M., Olivieri, A., Fornarino, S., et al. (2005). Saami and Berbers-An Unexpected Mitochondrial DNA Link. Am. J. Hum. Genet. 76 (5), 883-886. doi:10.1086/430073

Achilli, A., Rengo, C., Magri, C., Battaglia, V., Olivieri, A., Scozzari, R., et al. (2004). The Molecular Dissection of mtDNA Haplogroup H Confirms that the FrancoCantabrian Glacial Refuge Was a Major Source for the European Gene Pool. Am. J. Hum. Genet. 75 (5), 910-918. doi:10.1086/425590

Allentoft, M. E., Sikora, M., Sjögren, K.-G., Rasmussen, S., Rasmussen, M., Stenderup, J., et al. (2015). Population Genomics of Bronze Age Eurasia. Nature 522 (7555), 167-172. doi:10.1038/nature14507

An, C.-B., Chen, F.-H., and Barton, L. (2008). Holocene Environmental Changes in Mongolia: A Review. Glob. Planet. Change 63 (4), 283-289. doi:10.1016/ j.gloplacha.2008.03.007

Andrews, R. M., Kubacka, I., Chinnery, P. F., Lightowlers, R. N., Turnbull, D. M., and Howell, N. (1999). Reanalysis and Revision of the Cambridge Reference Sequence for Human Mitochondrial DNA. Nat. Genet. 23 (2), 147. doi:10.1038/ 13779

Bai, H., Guo, X., Narisu, N., Lan, T., Wu, Q., Xing, Y., et al. (2018). Whole-genome Sequencing of 175 Mongolians Uncovers Population-specific Genetic Architecture and Gene Flow throughout North and East Asia. Nat. Genet. 50 (12), 1696-1704. doi:10.1038/s41588-018-0250-5

Bouckaert, R., Vaughan, T. G., Barido-Sottani, J., Duchêne, S., Fourment, M., Gavryushkina, A., et al. (2019). BEAST 2.5: An Advanced Software Platform for Bayesian Evolutionary Analysis. Plos Comput. Biol. 15 (4), e1006650. doi:10.1371/journal.pcbi.1006650

Capodiferro, M. R., Aram, B., Raveane, A., Rambaldi Migliore, N., Colombo, G., Ongaro, L., et al. (2021). Archaeogenomic Distinctiveness of the Isthmo-Colombian Area. Cell 184 (7), 1706-1723. doi:10.1016/ j.cell.2021.02.040

Cavalli-Sforza, L. L., Menozzi, P., and Piazza, A. (1994). The History and Geography of Human Genes. Princeton NJ: Princeton University Press.

Chen, C., Li, Y., Tao, R., Jin, X., Guo, Y., Cui, W., et al. (2020). The Genetic Structure of Chinese Hui Ethnic Group Revealed by Complete Mitochondrial Genome Analyses Using Massively Parallel Sequencing. Genes 11 (11), 1352. doi:10.3390/genes11111352

Chikisheva, T. A., Gubina, M. A., Kulikov, I. V., Karafet, T. M., Voevoda, M. I., and Romaschenko, A. G. (2007). A Paleogenetic Study of the Prehistoric Populations of the Altai. Archeol. Ethnol. Anthropol. Eurasia 32, 130-142. doi:10.1134/S156301100704012

Comas, D., Calafell, F., Mateu, E., Pérez-Lezaun, A., Bosch, E., Martínez-Arias, R., et al. (1998). Trading Genes along the Silk Road: mtDNA Sequences and the Origin of central Asian Populations. Am. J. Hum. Genet. 63 (6), 1824-1838. doi:10.1086/302133

Derenko, M., Malyarchuk, B., Denisova, G., Perkova, M., Litvinov, A., Grzybowski, T., et al. (2014). Western Eurasian Ancestry in Modern Siberians Based on Mitogenomic Data. BMC Evol. Biol. 14, 217. doi:10.1186/s12862-014-0217-9

\section{ACKNOWLEDGMENTS}

We are grateful to all the volunteers who generously participated in this study and made this research possible.

\section{SUPPLEMENTARY MATERIAL}

The Supplementary Material for this article can be found online at: https://www.frontiersin.org/articles/10.3389/fgene.2021.819337/ full\#supplementary-material

Dür, A., Huber, N., and Parson, W. (2021). Fine-tuning Phylogenetic Alignment and Haplogrouping of mtDNA Sequences. Int. J. Mol. Sci. 22 (11), 5747. doi:10.3390/ijms22115747

Excoffier, L., and Lischer, H. E. L. (2010). Arlequin Suite Ver 3.5: a New Series of Programs to Perform Population Genetics Analyses under Linux and Windows. Mol. Ecol. Resour. 10 (3), 564-567. doi:10.1111/j.1755-0998.2010.02847.x

González-Ruiz, M., Santos, C., Jordana, X., Simón, M., Lalueza-Fox, C., Gigli, E., et al. (2012). Tracing the Origin of the East-West Population Admixture in the Altai Region (Central Asia). PloS one 7 (11), e48904. doi:10.1371/ journal.pone.0048904

Huber, N., Parson, W., and Dür, A. (2018). Next Generation Database Search Algorithm for Forensic Mitogenome Analyses. Forensic Sci. Int. Genet. 37, 204-214. doi:10.1016/j.fsigen.2018.09.001

Jeong, C., Balanovsky, O., Lukianova, E., Kahbatkyzy, N., Flegontov, P., Zaporozhchenko, V., et al. (2019). The Genetic History of Admixture across Inner Eurasia. Nat. Ecol. Evol. 3 (6), 966-976. doi:10.1038/s41559-019-0878-2

Jeong, C., Wang, K., Wilkin, S., Taylor, W. T. T., Miller, B. K., Bemmann, J. H., et al. (2020). A Dynamic 6,000-Year Genetic History of Eurasia's Eastern Steppe. Cell 183 (4), 890-904. doi:10.1016/j.cell.2020.10.015

Keyser, C., Bouakaze, C., Crubézy, E., Nikolaev, V. G., Montagnon, D., Reis, T., et al. (2009). Ancient DNA Provides New Insights into the History of South Siberian Kurgan People. Hum. Genet. 126 (3), 395-410. doi:10.1007/s00439009-0683-0

Keyser, C., Zvénigorosky, V., Gonzalez, A., Fausser, J.-L., Jagorel, F., Gérard, P., et al. (2021). Genetic Evidence Suggests a Sense of Family, Parity and Conquest in the Xiongnu Iron Age Nomads of Mongolia. Hum. Genet. 140 (2), 349-359. doi:10.1007/s00439-020-02209-4

Köstenbauer, J. (2017). Surgical Wisdom and Genghis Khan's Pax Mongolica. ANZ J. Surg. 87 (3), 116-120. doi:10.1111/ans.13813

Kovalev, A., and Erdenebaatar, D. (2009). "Discovery of New Cultures of the Bronze Age in Mongolia According to the Data Obtained by the International Central Asian Archaeological Expedition," in Current Archaeological Research in Mongolia. Editors J. Bemmann, H. Parzinger, E. Pohl, and D. Tseveendorj (Bonn, DE: University of Bonn).

Kumar, S., Stecher, G., Li, M., Knyaz, C., and Tamura, K. (2018). MEGA X: Molecular Evolutionary Genetics Analysis across Computing Platforms. Mol. Biol. Evol. 35 (6), 1547-1549. doi:10.1093/molbev/msy096

Lalueza-Fox, C., Sampietro, M. L., Gilbert, M. T. P., Castri, L., Facchini, F., Pettener, D., et al. (2004). Unravelling Migrations in the Steppe: Mitochondrial DNA Sequences from Ancient central Asians. Proc. R. Soc. Lond. B 271 (1542), 941-947. doi:10.1098/rspb.2004.2698

Lan, Q., Xie, T., Jin, X., Fang, Y., Mei, S., Yang, G., et al. (2019). MtDNA Polymorphism Analyses in the Chinese Mongolian Group: Efficiency Evaluation and Further Matrilineal Genetic Structure Exploration. Mol. Genet. Genomic Med. 7 (10), e00934. doi:10.1002/mgg3.934

Librado, P., and Rozas, J. (2009). DnaSP V5: a Software for Comprehensive Analysis of DNA Polymorphism Data. Bioinformatics 25 (11), 1451-1452. doi:10.1093/bioinformatics/btp187

Liu, X. (2010). The Silk Road in World History. New York: Oxford University Press.

Modi, A., Lancioni, H., Cardinali, I., Capodiferro, M. R., Rambaldi Migliore, N., Hussein, A., et al. (2020). The Mitogenome Portrait of Umbria in Central Italy as Depicted by Contemporary Inhabitants and Pre-roman Remains. Sci. Rep. 10 (1), 10700. doi:10.1038/s41598-020-67445-0 
Narasimhan, V. M., Patterson, N., Moorjani, P., Rohland, N., Bernardos, R., Mallick, S., et al. (2019). The Formation of Human Populations in South and Central Asia. Science 365 (6457), eaat7487. doi:10.1126/science.aat7487

Ning, C., Zheng, H.-X., Zhang, F., Wu, S., Li, C., Zhao, Y., et al. (2021). Ancient Mitochondrial Genomes Reveal Extensive Genetic Influence of the Steppe Pastoralists in Western Xinjiang. Front. Genet. 12, 740167. doi:10.3389/ fgene.2021.740167

Orkhonselenge, A., Komatsu, G., and Uuganzaya, M. (2018). Middle to Late Holocene Sedimentation Dynamics and Paleoclimatic Conditions in the Lake Ulaan basin, Southern Mongolia. Geomorphologie 24 (4), 351-363. doi:10.4000/ geomorphologie.12219

Ottoni, C., Primativo, G., Hooshiar Kashani, B., Achilli, A., Martínez-Labarga, C., Biondi, G., et al. (2010). Mitochondrial Haplogroup H1 in north Africa: an Early Holocene Arrival from Iberia. PloS one 5 (10), e13378. doi:10.1371/ journal.pone.0013378

Palstra, F. P., Heyer, E., and Austerlitz, F. (2015). Statistical Inference on Genetic Data Reveals the Complex Demographic History of Human Populations in central Asia. Mol. Biol. Evol. 32 (6), 1411-1424. doi:10.1093/molbev/msv030

Parson, W., and Dür, A. (2007). EMPOP-A Forensic mtDNA Database. Forensic Sci. Int. Genet. 1 (2), 88-92. doi:10.1016/j.fsigen.2007.01.018

Parson, W., Strobl, C., Huber, G., Zimmermann, B., Gomes, S. M., Souto, L., et al. (2013). Evaluation of Next Generation mtGenome Sequencing Using the Ion Torrent Personal Genome Machine (PGM). Forensic Sci. Int. Genet. 7 (5), 543-549. doi:10.1016/j.fsigen.2013.06.003

Perego, U. A., Lancioni, H., Tribaldos, M., Angerhofer, N., Ekins, J. E., Olivieri, A., et al. (2012). Decrypting the Mitochondrial Gene Pool of Modern Panamanians. Plos One 7 (6), e38337. doi:10.1371/journal.pone.0038337

Posth, C., Renaud, G., Mittnik, A., Drucker, D. G., Rougier, H., Cupillard, C., et al. (2016). Pleistocene Mitochondrial Genomes Suggest a Single Major Dispersal of Non-africans and a Late Glacial Population Turnover in Europe. Curr. Biol. 26 (6), 827-833. doi:10.1016/j.cub.2016.01.037

Pugach, I., Matveev, R., Spitsyn, V., Makarov, S., Novgorodov, I., Osakovsky, V., et al. (2016). The Complex Admixture History and Recent Southern Origins of Siberian Populations. Mol. Biol. Evol. 33 (7), 1777-1795. doi:10.1093/molbev/ msw055

Quintana-Murci, L., Chaix, R., Wells, R. S., Behar, D. M., Sayar, H., Scozzari, R., et al. (2004). Where West Meets East: the Complex mtDNA Landscape of the Southwest and Central Asian Corridor. Am. J. Hum. Genet. 74 (5), 827-845. doi: $10.1086 / 383236$

R Core Team (2021). R: A Language and Environment for Statistical Computing. Vienna, Austria: R Foundation for Statistical Computing.

Ricaut, F.-X., Keyser-Tracqui, C., Bourgeois, J., Crubézy, E., and Ludes, B. (2004a). Genetic Analysis of a Scytho-Siberian Skeleton and its Implications for Ancient Central Asian Migrations. Hum. Biol. 76 (1), 109-125. doi:10.1353/ hub.2004.0025

Ricaut, F.-X., Keyser-Tracqui, C., Cammaert, L., Crubézy, E., and Ludes, B. (2004b). Genetic Analysis and Ethnic Affinities from Two Scytho-Siberian Skeletons. Am. J. Phys. Anthropol. 123 (4), 351-360. doi:10.1002/ajpa.10323

Rogers, L. L., Honeychurch, W., Amartuvshin, C., and Kaestle, F. A. (2019). U5a1 Mitochondrial DNA Haplotype Identified in Eneolithic Skeleton from Shatar Chuluu, Mongolia. Hum. Biol. 91 (4), 213-223. doi:10.13110/ humanbiology.91.4.01

Rozas, J., Ferrer-Mata, A., Sánchez-DelBarrio, J. C., Guirao-Rico, S., Librado, P., Ramos-Onsins, S. E., et al. (2017). DnaSP 6: DNA Sequence Polymorphism Analysis of Large Data Sets. Mol. Biol. Evol. 34 (12), 3299-3302. doi:10.1093/ molbev/msx 248

Schurr, T., and Pipes, L. (2011). "Prehistory of Mongolian Populations as Revealed by Studies of Osteological, Dental, and Genetic Variation," in Mapping Mongolia: Situating Mongolia in the World from Geologic Time to the Present. Editor P. L. W. Sabloff (Philadelphia: University of Pennsylvania Museum Press), 125-165.

Soares, P., Ermini, L., Thomson, N., Mormina, M., Rito, T., Röhl, A., et al. (2009). Correcting for Purifying Selection: an Improved Human Mitochondrial
Molecular Clock. Am. J. Hum. Genet. 84 (6), 740-759. doi:10.1016/ j.ajhg.2009.05.001

Strobl, C., Eduardoff, M., Bus, M. M., Allen, M., and Parson, W. (2018). Evaluation of the Precision ID Whole MtDNA Genome Panel for Forensic Analyses. Forensic Sci. Int. Genet. 35, 21-25. doi:10.1016/ j.fsigen.2018.03.013

Thorvaldsdottir, H., Robinson, J. T., and Mesirov, J. P. (2013). Integrative Genomics Viewer (IGV): High-Performance Genomics Data Visualization and Exploration. Brief Bioinform. 14 (2), 178-192. doi:10.1093/bib/bbs017

van Oven, M., and Kayser, M. (2009). Updated Comprehensive Phylogenetic Tree of Global Human Mitochondrial DNA Variation. Hum. Mutat. 30 (2), E386-E394. doi:10.1002/humu.20921

Wang, C. C., Yeh, H. Y., Popov, A. N., Zhang, H. Q., Matsumura, H., Sirak, K., et al. (2021). Genomic Insights into the Formation of Human Populations in East Asia. Nature 591 (7850), 413-419. doi:10.1038/s41586-021-03336-2

Wang, W., Ding, M., Gardner, J. D., Wang, Y., Miao, B., Guo, W., et al. (2021). Ancient Xinjiang Mitogenomes Reveal Intense Admixture with High Genetic Diversity. Sci. Adv. 7 (14), eabd6690. doi:10.1126/sciadv.abd6690

Weissensteiner, H., Pacher, D., Kloss-Brandstätter, A., Forer, L., Specht, G., Bandelt, H.-J., et al. (2016). HaploGrep 2: Mitochondrial Haplogroup Classification in the Era of High-Throughput Sequencing. Nucleic Acids Res. 44 (W1), W58-W63. doi:10.1093/nar/gkw233

Wells, R. S., Yuldasheva, N., Ruzibakiev, R., Underhill, P. A., Evseeva, I., BlueSmith, J., et al. (2001). The Eurasian Heartland: a continental Perspective on Y-Chromosome Diversity. Proc. Natl. Acad. Sci. 98 (18), 10244-10249. doi:10.1073/pnas.171305098

Wilkin, S., Ventresca Miller, A., Fernandes, R., Spengler, R., Taylor, W. T.-T., Brown, D. R., et al. (2021). Dairying Enabled Early Bronze Age Yamnaya Steppe Expansions. Nature 598, 629-633. doi:10.1038/s41586-021-03798-4

Yang, L., Tan, S., Yu, H., Zheng, B., Qiao, E., Dong, Y., et al. (2008). Gene Admixture in Ethnic Populations in Upper Part of Silk Road Revealed by mtDNA Polymorphism. Sci. China Ser. C 51 (5), 435-444. doi:10.1007/s11427008-0056-2

Yao, Y.-G., Kong, Q. P., Wang, C. Y., Zhu, C. L., and Zhang, Y. P. (2004). Different Matrilineal Contributions to Genetic Structure of Ethnic Groups in the Silk Road Region in China. Mol. Biol. Evol. 21 (12), 2265-2280. doi:10.1093/molbev/ msh238

Yunusbayev, B., Metspalu, M., Metspalu, E., Valeev, A., Litvinov, S., Valiev, R., et al. (2015). The Genetic Legacy of the Expansion of Turkic-Speaking Nomads across Eurasia. Plos Genet. 11 (4), e1005068. doi:10.1371/journal.pgen.1005068

Zerjal, T., Xue, Y., Bertorelle, G., Wells, R. S., Bao, W., Zhu, S., et al. (2003). The Genetic Legacy of the Mongols. Am. J. Hum. Genet. 72 (3), 717-721. doi:10.1086/367774

Conflict of Interest: The authors declare that the research was conducted in the absence of any commercial or financial relationships that could be construed as a potential conflict of interest.

Publisher's Note: All claims expressed in this article are solely those of the authors and do not necessarily represent those of their affiliated organizations, or those of the publisher, the editors, and the reviewers. Any product that may be evaluated in this article, or claim that may be made by its manufacturer, is not guaranteed or endorsed by the publisher.

Copyright (c) 2022 Cardinali, Bodner, Capodiferro, Amory, Rambaldi Migliore, Gomez, Myagmar, Dashzeveg, Carano, Woodward, Parson, Perego, Lancioni and Achilli. This is an open-access article distributed under the terms of the Creative Commons Attribution License (CC BY). The use, distribution or reproduction in other forums is permitted, provided the original author(s) and the copyright owner(s) are credited and that the original publication in this journal is cited, in accordance with accepted academic practice. No use, distribution or reproduction is permitted which does not comply with these terms. 